\title{
Análisis crítico de ensayos clínicos aleatorizados: Riesgo de sesgo
}

\author{
Critical analysis of randomized clinical trials: The risk of bias \\ Marco Alarcón Palacios ${ }^{1, a, b}$, Roberto Carlos Ojeda Gómez ${ }^{1, c . d}$, Iveht Lucy Ticse Huaricancha ${ }^{1, c, d}$, Katherine \\ Cajachagua Hilario ${ }^{1, c, d}$.
}

\section{RESUMEN}

Los ensayos clínicos aleatorizados (ECAs) presentan el nivel de investigación experimental más alto según los niveles de evidencia. Los resultados encontrados en ellos brindarán las pautas para la elección del mejor tratamiento clínico en los pacientes, es por ello la necesidad de conocer cuáles son las pautas para analizar críticamente los ECAs en la búsqueda de la precisión científica. El 'Riesgo de Sesgo' es una herramienta desarrollada por la "Cochrane Collaboration” para evaluar la metodología de la evidencia científica siendo útil en revisiones sistemáticas para el análisis individual de los ECAs incluidos. La presente revisión busca desarrollar los conceptos del 'Riesgo de Sesgo' definiendo cada uno de sus componentes para el correcto análisis de los artículos científicos.

PALABRAS CLAVE: Sesgo, ensayo clínico, validez, distribución aleatoria, revisión, método doble ciego.

\section{SUMMARY}

Randomized clinical trials (RCTs) represent the highest level of experimental research according to levels of evidence. The findings of RCTs provide guidelines for choosing the best clinical treatment. Reason why it is a need to critically assess their methodology in order to show the accuracy.'Risk of Bias' is a tool developed by the Cochrane Collaboration to determine the quality of evidence in systematic reviews to be reliable for the individual analysis of the included RCTs. The present review aims to present the concepts of risk bias defining each of its items for the proper assessment of scientific articles.

KEYWORDS: Risk, bias, clinical trial, validity, random allocation, review, double-blind method.

Facultad de Estomatología, Universidad Peruana Cayetano Heredia. Lima, Perú

Magister en Estomatología

Especialista en Periodoncia

Cirujano Dentista

Maestrando. 


\section{INTRODUCCIÓN}

Los ensayos clíncos aleatorizados (ECAs) representan la mejor evidencia científica para la toma de decisiones clínicas en odontología. El desarrollo científico reflejado en la creciente producción de artículos originales en diferentes revistas nos lleva a ser minuciosos en el análisis de las publicaciones para encontrar las mejores evidencias disponibles. Así pues resulta importante realizar una evaluación de la validez interna de cada artículo haciendo énfasis en el 'Riesgo de Sesgo' de los ECAs (1).

La colaboración Cochrane nos brinda una herramienta específica para evaluar el riesgo de sesgo de los ECAs. Para esto incluye una descripción y valoración para cada ítem en una tabla de "Riesgo de Sesgo" el cual nos ofrece una evaluación final que determina la calidad del artículo $(1,2)$.

El objetivo de la presente revisión fue describir y analizar el riesgo de sesgos de ensayos clínicos a través del Cochrane Handbook for Systematic Review for Interventions del año 2008.

\section{Sesgo y Validez}

Por definición, "Sesgo es un error sistemático o una desviación de la verdad en los resultados"(1). Esta desviación del valor verdad puede dar como resultado la sub/sobre-estimación de los efectos de una determinada intervención (2); sin embargo no es posible conocer hasta que grado han afectado los sesgos de los resultados en un estudio específico por ello es más apropiado considerar el "Riesgo de Sesgo"(1), que es una herramienta utilizada para poder evaluar la validez de los ECAs (3) y poder analizar si los resultados de un estudio pueden ser interpretados confiablemente (1). La validez se define como la capacidad del instrumento para medirlo que se cree que está midiendo, es decir sería la evaluación de la precisión con la que un instrumento mide correctamente lo que desea medir (5-7).

\section{Fuentes de sesgos en los ensayos clínicos}

En el 2008, The Cochrane Collaboration publicó una nueva herramienta muy útil para evaluar el riesgo de sesgo en los ensayos clínicos aleatorizados (3), planteando seis niveles de sesgo:
1. Sesgos de selección. Son las diferencias sistemáticas entre las características iniciales de los grupos que se comparan, el cual debería tener un método adecuado para asignar las intervenciones y está sería basada en algún proceso al azar (aleatorio). De esta manera todos los participantes en el estudio tienen la misma oportunidad de ser asignados a cada uno de los grupos, esto es llamado "Generación de Secuencia”. Por otro lado también se debería ocultar las asignaciones de quienes están involucrados en el reclutamiento del estudio, esto es llamado: “Ocultamiento de la Asignación” $(1,2)$.

2. Sesgos de realización. Son las diferencias sistemáticas entre grupos, posterior al reclutamiento, en lo referente al enmascaramiento de los participantes y los operadores. El sesgo de realización minimiza el sesgo (8), ya que podría reducir el riesgo de que el participante o el operador al tener conocimiento de que intervención recibió. Ciertamente, como en el caso de ensayo de biomateriales, particularmente en odontologia,hay que considerar que el enmascaramiento del operador y examinador en ocasiones es poco fiable (aplicable también para sesgo de detección) $(1,9)$.

3. Sesgos de detección. Son las diferencias sistemáticas entre grupos referido al cegamiento de quiénes evaluarón los resultados de las intervenciones. En estos casos es necesario que el evaluador de los resultados ya sea clínico, radiológico o histológico no tenga el conocimiento de cuál fue la intervención recibida por cada uno de los grupos $(1,9)$.

4. Sesgos de desgaste. Son las diferencias sistemáticas entre grupos debido a abandonos, que pueden ser voluntarios o involuntarios, observándose que los grupos se encuentren incompletos al final de la realización los ensayos clínicos. Un ejemplo son las exclusiones en donde algunos participantes son omitidos de los análisis, a pesar de que los datos sobre sus resultados están disponibles $(1,10)$.

5. Sesgo denotificación. Sonlas diferencias sistemáticas entre los resultados presentados y los no presentados. En un estudio publicado deben de ser presentados resultados acordes con los objetivos planteados al inicio del curso. En muchos casos los investigadores tienden a variar el enfoque de los estudios debido a que los resultados no salieron tal como se esperaban (11). 
Tabla 1. Herramienta de la Colaboracion Cochrane para evaluar el riesgo de sesgo

Dominio

Sesgo de selección

Sesgo de realización

Sesgo de detección.

Sesgo de desgaste.

Sesgo de notificación

Otros sesgos

Otras fuentes
sesgo.
Generación de la Secuencia.

Ocultamiento de la asignación

Cegamiento de los participantes y del personal.

Cegamiento de los evaluadores del resultado.

Datos de resultado Incompletos.

\section{Descripción}

Describe el método utilizado para generar la secuencia de asignación con detalle suficiente para permitir una evaluación de si la misma produjo grupos comparables.

Describe el método utilizado para ocultar la secuencia de asignación con detalle suficiente para determinar si las asignaciones a la intervención se podían prever antes o durante el reclutamiento.

Valoración de los revisores

Asignación sesgada a las intervenciones. A causa de una generación inadecauda de la secuencia de aleatorización.

Asignación sesgada a las intervenciones. A causa de una ocultación inadecuada de las asignaciones antes de asignarlas.

Describe todas las medidas utilizadas, si se utilizó alguna, para cegar a los participantes y al personal del estudio, al conocimiento de qué intervención recibió un participante.

Proporcionar cualquier información con respecto a si el cegamiento propuesto fue efectivo.

Describe todas las medidas utilizadas, si se utilizó alguna, para cegar a los evaluadores del resultado del estudio al conocimiento de qué intervención recibió un participante.

Proporcionar cualquier información con respecto a si el cegamiento propuesto fue efectivo.

Describe la compleción de los datos de resul- A causa de la cantidad, la tado para cada resultado principal, incluidos los abandonos y las exclusiones del análisis. Señalar si se describieron los abandonos y las exclusiones, los números en cada grupo de intervención (comparados con el total de participantes asignados al azar), los motivos de las deserciones /exclusiones cuando se detallaron, y cualquier reinclusión en los análisis realizada por los revisores.

Notificación selecti- Señala cómo los revisores examinaron la posiva de los resultados.

bilidad de la notificación selectiva de los resultados, y qué encontraron.

de la

notificación selectiva de los resultados.

Sesgo debido a otros problemas no abordados en los apartados instrumento.

Si en el protocolo de la revisión se especificaron preguntas/ítems particulares, se deberían proporcionar las respuestas para cada pregunta/ítem. naturaleza o el manejo de los datos de resultado incompletos.
A causa del conocimiento por parte de los participany del personal durante el asignadas.

A causa del conocimiento por parte de los evaluadores de los resultados de las intervenciones asignadas. 
6. Otros sesgos. Se pueden encontrar en circunstancias específicas sesgos de contaminación, en la cual las intervenciones del grupo experimental y el control "se mezclan", sesgos en la elección de la pregunta que el ensayo tiene la intención de responder, sesgos de diseño incorrecto (2) así como sesgos que solamente se encuentran en un ámbito clínico determinado (1).

En la tabla 1 se hace una presentación resumida de los diferentes sesgos, su descripción y la valoración que se le debiera dar a la evaluación.

\section{Evaluación del Riesgo de Sesgo}

Cada uno de los seis items del riesgo de sesgo debe ser evaluado individualmente en los ECAs. Luego de ello se asignará un valor que determinará el alto o bajo nivel de sesgo. Existe la posibilidad de definir el sesgo como no claro, en estas situaciones se debería tomar contacto con el autor del artículo para esclarecer el nivel de sesgo (Tabla 2) (1-3).

Tabla 2. Evaluacióndel riesgo de sesgo

Riesgo de Interpretación
sesgo

\begin{tabular}{ll}
\hline Bajo riesgo de & Sesgo poco probable que altere \\
Sesgo & significativamente los resultados.
\end{tabular}

Riesgo de sesgo poco claro

Alto riesgo de sesgo
Sesgo que hace surgir algunas dudas acerca de los resultados.

Sesgo que debilita seriamente la confianza en losr esultados.

\section{DISCUSIÓN}

Resulta claro que no todas las publicaciones científicas cuentan con la validez respectiva para servir como guía clínica en la atención de los pacientes. En el presente artículo se hizo una revisión sobre los sesgos de las investigaciones basados en el Manual de Cochrane para intervenciones buscando evaluar la calidad y validez de ECAs mediante el 'Riesgo de Sesgo; describiéndose cada ítem para la valoración del riesgo de sesgo de una manera más concreta, para así poder minimizar los sesgos en los futuros trabajos de investigación $(1,2)$.

El primer objetivo de la lectura crítica de un artículo de investigación consiste en determinar correctamente el diseño de la investigación científica. La diferencia entre un ensayo clínico aleatorizado y un ensayo clínico controlado es la distribución al azar de ambos grupos que van a recibir el tratamiento. Este primer punto de evaluación resulta clave y sumamente importante para valorar el nivel de la evidencia científica.

Un segundo ítem a considerar importante en la evaluación del artículo científico es el ocultamiento de la asiganción de los grupos, es decir tratar de proteger la aleatorización mediante sobres cerrados, oscuros y numerados secuencialmente que hará que el conocimiento de que tratamiento recibirá cada grupo sea sabido en el mismo día de la intervención.

Un tercer punto a considerar el cegamiento tanto de los pacientes, operadores, evaluadores de los resultados y estadísticos. Si bien en muchos estudios resulta dificil hacer el enmascaramiento para todos los grupos el lector debiera identificar que se hizo el intento para catalogarlo como un bajo riesgo de sesgo.

El siguiente punto a considerar en la evaluación del sesgo de un ensayo clínico es la cantidad de abandonos que surgieron en la investigación, los cuales debieran estar reportados. Es sabido que en algunas situaciones los investigadores pueden ocultar la información de ciertos resultados o tener menos muestra que al inicio 
por el fracaso del tratamiento experimental.

Finalmente un punto muy importante a considerar es el reporte selectivo de la información el cual se refiere a que el investigador manipula a su conveniencia los resultados encontrados lo cuales no son los mismos planteados al inicio dela investigación, los cuales debieran haber sido registrados al momento de iniciar el proyecto.

Por todo lo antes mencionado la fiabilidad de los resultados en los ensayos clínicos dependerá en gran medida de la evaluación de riesgo de sesgos. A través de los años se han desarrollado guías y propuestas para desarrollar análisis crítico de la literatura científica (12-14). Un correcto entendimiento de sus componentes determinará un mejor plantemiento al momento de diseñar un estudio experimental en humanos así como para los lectores servirá para una mejor toma de decisiones clínicas en nuestros pacientes.

\section{Correspondencia:}

Dr. Marco Alarcón Palacios

Telefono: 511-4635959 / Celular: 51-999635259

\section{REFERENCIAS BIBLIOGRÁFICAS}

1. Higgins JPT, Green S. (editors).Cochrane Handbook for Systematic Reviews of Interventions. Version 5.1.0 updated March 2011. The Cochrane Collaboration; 2011.p. 197-255. (Citado el 15 setiembre del 2015) Disponible en: http://handbook.cochrane.org/

2. Higgins JPT, Green S (editors). Cochrane Handbook for Systematic Reviews of Interventions Version 5.1.0 [updated March 2011]. The Cochrane Collaboration; 2011. Available from www.cochrane-handbook.org..

3. Hartling L, Bond K, Vandermeer B, Seida J, Dryden $\mathrm{D}$, Rowe B. Applying the risk of bias tool in a systematic review of combination long-acting beta-agonists and inhaled corticosteroids for persistent asthma. PLoSOne. 2011; 6(2):24-30.

4. Moher D, Jadad AR, Nichol G, Penman M, Tugwell $\mathrm{P}$, Walsh S. Assessing the quality of randomized controlled trials: an annotated bibliography of scales and checklists. Control Clin Trials. 1995;16(1):62-73.

5. Jadad A, Moore A, Carroll D, et al. Assessing the quality of reports of randomized clinical trials: Is Blinding Necessary?. Control Clin Trials. 1996; 17(1):1-12.

6. Higgins J, Altman D, Gotzsche P, et al. The Cochrane
Collaboration's tool for assessing risk of bias in randomisedtrials.BMJ 2011;59 (28):1-9

7. Berlin JA. Does blinding of readers affect the results of meta-analyses? University of Pennsylvania Meta-analysis Blinding Study Group. Lancet. 1997; 350(9072): 185-186.

8. 8. Haahr MT, Hróbjartsson A. Who is blinded in randomized clinical trials? A study of 200 trials and a survey of authors. Clinical Trials. 2006;3(4):360-365.

9. Safer DJ. Design and reporting modifications in industry-sponsored comparative psychopharmacology trials. J Nerv Ment Dis. 2002;190(9):583-92.

10. Chan AW, Krleža-Jeric K, Schmid I, Altman DG. Outcome reporting bias in randomized trials funded by the Canadian Institutes of Health Research. CMAJ. 2004; 171(7): 735-740.

11. Chan AW, Altman DG. Identifying outcome reporting bias in randomised trials on PubMed: review of publications and survey of authors. BMJ 2005; 330 (7494): 753- 759

12. Moher D, Cook DJ, Eastwood S, Olkin I, Rennie D, Stroup DF. Improving the quality of reports of meta -analyses of randomised controlled trials: the QUOROM statement. Quality of Reporting of Meta-analyses. Lancet. 1999;354(9193):1896-1900.

13. Shaw RL, Booth A, Sutton AJ, et al. Finding qualitative research: an evaluation of search strategies. BMCMedical research methodology 2004;4(1):1-5.

14. Faggion CM Jr, Listl S, Alarcón MA. Is the evaluation of risk of bias in periodontology and implant dentistry comprehensive? A systematic review. J Clin Periodontol. 2015 ;42(5):488-94.

Recibido : 15/01/2015

Aceptado: 16/04/2015 